\title{
Time Lapse between Initial Stroke Symptoms to Performance of CT Scan in Patients with Ischemic Stroke in Pakistan
}

\author{
Shahzad Ahmed Daula ${ }^{1}$, Muhammad Ans ${ }^{2}$, Muhammad Uzair ${ }^{3}$, Dr. Zareen Fatima ${ }^{4}$, Dr. Ahmed Bilal Waqar ${ }^{5}$ \\ ${ }^{1,3}$ Faculty of Allied Health Sciences, University of Lahore, 1 - KM Defence Road, Lahore, Pakistan
}

${ }^{2}$ Department of Radiological Sciences and Medical Imaging, Faculty of Health and Allied Sciences, Imperial College of Business Studies, City Campus, 25-B, Lower Mall Road Lahore, Pakistan.

${ }^{3}$ Faculty of Health and Allied Sciences, Imperial College of Business Studies, City Campus, 25-B, Lower Mall Road Lahore, Pakistan.

${ }^{4}$ MBBS, MS, PhD, Department of Radiological Sciences and Medical Imaging, Faculty of Health and Allied Sciences, Imperial College of Business Studies, City Campus, 25-B, Lower Mall Road Lahore, Pakistan.

${ }^{5}$ MBBS, PhD, Faculty of Health and Allied Sciences, Imperial College of Business Studies, City Campus, 25-B, Lower Mall Road Lahore, Pakistan

\begin{abstract}
Objective: To determine time lapse between first symptom to CT scan in stroke patients, in Lahore Pakistan. Methods: Study was conducted at a local health facility in Lahore, Pakistan on 30 patients with confirmed ischemic stroke on CT. Results: Time lapse ranged between 60-1200min, mean: 250min. Factors that affected this duration were knowledge of the symptoms, patient's socioeconomic status andeducational level, distance from medical facility and availability of $C T$ scanner in medical facilities and transportation services. Conclusion: Increasing knowledge of stroke symptoms and installation of CT scanners are urgently required in Pakistanto avoid permanent disabilities and mortality.
\end{abstract}

Keywords: Computed Tomography, delay, stroke

\section{Introduction}

Stroke remains one of the most important causes of disability and death in developing as well as developed countries. [1] Early recognition of its symptoms and timely therapeutic interventions has been reported to be very important in reducing both morbidity and mortality. $[2,3]$

Like many advancements in the field of medicine, reperfusion therapy comprising of recombinant tissue plasminogen activator or alteplasehas also revolutionized the treatment strategies of stroke. The efficiency of this treatment and hence the chances of improvement in outcome decrease as there is increased time lapse between the occurrence of stroke and administration of treatment.[3,4] Inspite of the availability of the treatment many cases of stroke do not get benefitted from it due to the time delay that lies between the occurrence of stroke to its proper diagnosis and identification. [5] In cases of ischemic stroke due to arterial occlusion there is region of brain parenchyma termed as "ischemic penumbra" that is threatened but remains viable for some time called as "therapeutic time window". [6] Best therapeutic results are noted within 90 minutes and reperfusion treatment is not performed after therapeutic time window of three hours after onset of the symptoms of stroke. [3]In addition early diagnosis and thus start of treatment and maintenance of patient's general condition are also reported to be very important in preventing complications such as pneumonia and favourable outcomes. [7]

Unenhanced computed tomography (CT) of the brain is the primary imaging modality used in the exclusion of intracranial hemorrhage and confirmation of ischemic stroke. [8-10]

\section{Materials and Methods}

This cross-sectional observational study was conducted after approval from Institutional Review Board for ethical issues. Informed consent was taken from the included patients.

The study was conducted for a period of 2 months at a local health facility in Lahore, Pakistan. 30 patients with an age range of 22-80 years and mean age of 62.1 years with confirmedischemic stroke on CT scan later on were included in the study. 17/30(56.7\%) were males while $13 / 30(43.3 \%)$ were females. Exclusion criteria were inability to tell the time of onset of symptoms, inability to communicate with no family member available to provide information, and refusal to provide informed consent.

The details about the patients symptoms, time of their initial appearance and factors causing the delay if any to reach the medical facility for $\mathrm{CT}$ scan were inquired either from the patient or attendant of the patient. The time of CT scan conduction was noted and then time lapse between appearance of first symptom and scan was calculated. The data were recorded on pre-designed data collection sheets. MedCalc software was used for statistical analysis of the data and generation of graphs. 


\section{International Journal of Science and Research (IJSR) \\ ISSN (Online): 2319-7064 \\ Index Copernicus Value (2013): 6.14 | Impact Factor (2015): 6.391}

\section{Results}

The time lapse between first symptoms of stroke to the performance of CT scan and confirmation of the diagnosis of stroke was found to be ranging from 60-1200 minutes with a median of $150 \mathrm{~min} .21 / 30(70 \%)$ were scanned before while $9 / 30(30 \%)$ got the scan done after 3 hrs of the stroke. However only $8 / 30(26.66 \%)$ got scanned within 90 minutes and $22 / 30(73.33 \%$ ) after this time. There was no significant statistical difference in the time delay in these patients based on gender or age $(\mathrm{r}=-.053)$.

The identified delays were noted to be due to multiple factors including: (1)Patient factors, which included lack of education, poor socio-economic status and fear of expenses which make them hesitant to seek medical attention. Lack of knowledge, inability to recognize and thus failure of reacting to the symptoms of stroke was also found to be a major risk factor in delay. (2) Factors related to medical services, i.e. distance from the nearest medical facility and availability of CT scanner in the medical facilities was also found to be very important in causing delay. (3) The inadequacy of transportation services and the traffic jams on the way to hospital also hindered the early and prompt diagnosis in patients with stroke.

\section{Discussion}

Our results confirmed previously reported factors claimed to be associated with the delays caused in the diagnosis of stroke although no other study reported these findings in context of CT confirmation of stroke diagnosis. Siddiqui M et al. [3] reports the median delay of about $6 \mathrm{hrs}$ in hospital arrival after the stroke. We however found a median delay of only 150 minutes in patients getting the CT scan done. The results although differ widely but the discrepancy can be explained on the basis of the fact that the study was conducted in 2008, the cause of relatively early arrival of patient to the medical facility and getting scanned might be due to the reason in improvement both in patient's knowledge as well as increased medical facilities since then. Another factor might be that the mentioned study was conducted in Karachi which is a bigger city compared to Lahore with even more population and far distances than in Lahore.

The main factors in the delay were identified to be the patient's unawareness of the symptoms of stroke and importance of early treatment. This also corresponds well to the observations made earlier. [5]

In a previous study history of previous stroke has been implicated as a factor in shorter delay while accompanied diabetes has been associated with a longer delay of hospital arrival in patients with diabetes. [11] We however admit the limitation of study not to have recorded such history in all of our patients.

We didn't find any statistically significant difference in the time delay between male and female population which differs from results of a previous study which states that there is more delay in females due to prolonged decision time compared to male patients. ${ }^{11}$ The results are however in accordance with Kim et al. who also did not find any association of prehospital delay with age or sex. [12]

Elderly population living alone in developed countries like Japan is also at a greater risk of prehospital delay such as described by Yanagida T et al. [7]No such factor was found in our study as a cause in our population. This difference is due to close family bonds and combined family systems in our society.

\section{Conclusion}

Programs for increasing awareness about the stroke symptoms and risk factors should be started on both Government and private levels. In addition medical facilities with installed CT scanners are urgently required in order to avoid permanent disabilities and mortality in many cases.

\section{References}

[1] Mellor RM, Bailey S, Sheppard J, Carr P, Quinn T, et al. Decisions and delays within stroke patients' route to the hospital: A qualitative study. Ann Emerg Med. 2015;65:279-287.

[2] Lacy RC, Suh DC, Bueno M, Kostis JB. Delay in presentation and evaluation for acute stroke. Stroke time registry for outcomes knowledge and epidemiology(S.T.R.O.K.E.). Stroke 2001; 32:63-69

[3] Siddiqui M, Siddiqui SR, Zafar A, Khan FS. Factors delaying hospital arrival of patients with acute stroke. J Pak Med Assoc 2008; 58(4): 178-181

[4] Saver JL. Improving reperfusion therapy for acute ischaemic stroke. Journal of Thrombosis and Haemostasis 2011; 9: 333-343

[5] Mandelzweig L, Goldbourt U, Boyko V, Tanne D. Perceptual, social, and behavioral factors associated with delays in seeking medical care in patients with symptoms of acute stroke. Stroke 2006; 37: 1248-1253

[6] Mahmood A, Sharif MA, Ali UZ, Khan MN. Time to hospital evaluation in patients of acute stroke for Alteplase therapy. RMJ. 2009; 34(1): 43-46

[7] Yanagida T, Fujimoto S, Inoue T, Suzuki S. Causes of prehospital delay in stroke patients in urban aging society. J ClinGerontolGeriatr 2014; 5: 77-81

[8] von Kummer R, Meyding-Lamade U, Forsting M, et al. Sensitivity and prognostic value of early $\mathrm{CT}$ in occlusion of the middle cerebral artery trunk. AJNR Am J Neuroradiol 1994; 15:9-15.

[9] Bahn MM, Oser AB, Cross DT, 3rd. CT and MRI of stroke. J MagnReson Imaging 1996; 6:833-845.

[10]von Kummer R, Nolte PN, Schnittger $H$, et al. Detecability of cerebral hemisphere ischaemic infarcts by CT within 6 hours of stroke. Neuroradiology 1996; 38:31-33

[11] Herlitz J, Wireklintsundström B, Bang A, Berglund A, Svennson L, Blomstrand C. Early identification and delay to treatment in myocardial infarction and stroke: differences and similarities. Scand. J. Trauma Resusc. Emerg. Med. 2010;18

[12] Kim YS, Park SS, Bae HJ, Cho AH, Cho YJ, et.al. Stroke awareness decreases prehospital delay after acute ischemic stroke in Korea. BMC Neurology 2011, 11:2.

\section{Volume 5 Issue 8, August 2016}




\section{Figures}

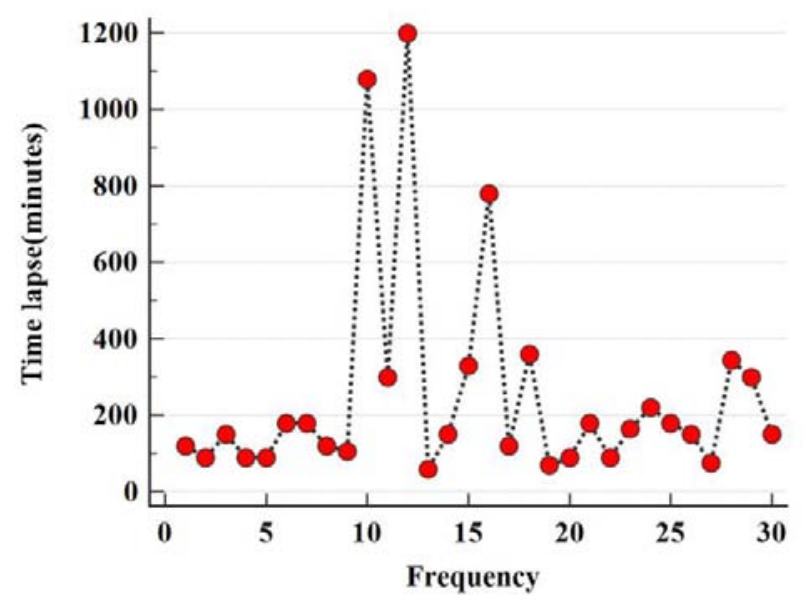

Figure 1

Figure 1 shows the distribution of time delay between the appearances of initial symptoms of stroke to the CT scan of brain in sample population.

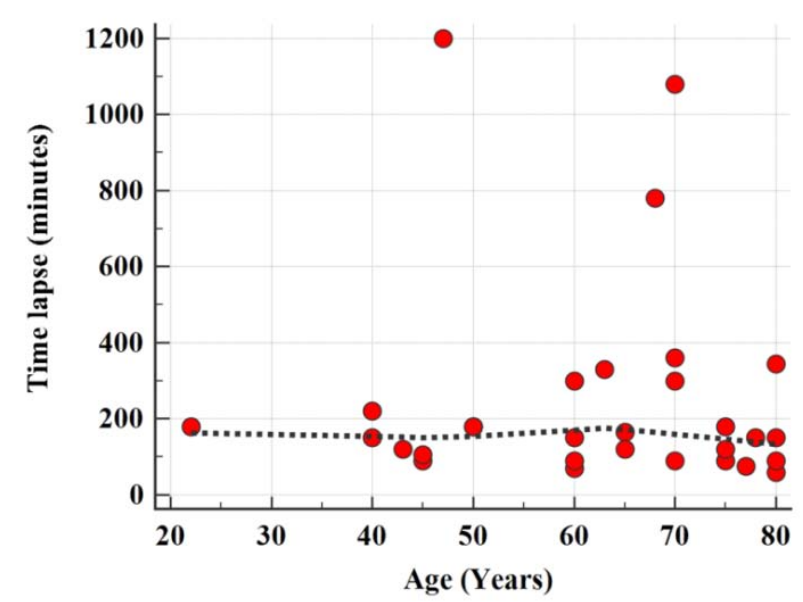

Figure 2

Figure 2 shows the distribution of time delay taken between the appearances of initial symptom of stroke to the CT scan of brain in sample population in relation to different ages in sample population. 